\title{
Sozialraumorientierung der Kinder- und Jugendhilfe
}

\section{Raumanalysen für die Annäherung von Lebenswelt und Hilfesystem}

MICHAEL NOACK

Michael Noack ist Krankenpfleger, Sozialarbeiter und wissenschaftlicher Mitarbeiter am Forschungsschwerpunkt "sozial raum management" an der Fachhochschule Köln und Lehrbeauftragter am Institut für Stadtteilentwicklung, Sozialraumorientierte Arbeit und Beratung (ISSAB) der Universität Duisburg-Essen.

Michael.Noack@fh-koeln.de

\author{
Der "Sozialraum" ist aus der Sozialen Arbeit nicht \\ mehr wegzudenken. Welche Bedeutung dabei \\ Sozialraumanalysen zukommt, kann am Beispiel \\ der Kinder- und Jugendhilfe gezeigt werden.
}

Um die notwendige Brücke zwischen den Lebenswelten junger Menschen sowie ihren Familien und dem Jugendhilfesystem bauen zu können, wird ein Raumbegriff benötigt, der sowohl im institutionellen Hilfesystem als auch in den verinselten Lebenswelten anschlussfähig ist (vgl. Fehren 2008: 154).

Daher wurde der Sozialraumorientierung ein Raumbegriff zugrunde gelegt, der einen Mittelweg zwischen beiden Sphären beschreitet, um sie als Einheit zu denken: "Je nach subjektiver Definition, Ausstattung und Gruppenzugehörigkeit werden also höchst individuell Sozialräume definiert. Gleichzeitig gibt es Überlappungen, an denen sich verschiedene höchst individuelle Sozialräume überschneiden. (...) Zum anderen wird der Sozialraum als Steuerungsgröße genutzt, definiert von Institutionen, die bezogen auf ein Wohngebiet Personal und Geldströme konzentrieren. Diese großen räumlichen Einheiten erfassen nie präzise die zahlreichen und individuellen Definitionen von Sozialräumen, sie werden jedoch sinnvollerweise geschnitten anhand möglichst plausibler und nachvollziehbarer Gebietsdefinitionen und sind somit Bindeglied zwischen der verwaltungsseits notwendigen Ordnungskategorie einerseits und den lebensweltlichen vorgenommenen Raumdefinitionen andererseits." (Hinte, Treß 2007: 32)

Sozialraumorientierung reduziert sich »nicht auf geometrische Beziehungen, die wir festsetzen, als wenn wir selbst, auf die Rolle des Zuschauers beschränkt, uns außerhalb des Raums befänden «, sondern »begreift Raum als das Ergebnis sozialen und organisatorischen Handelns, das den Handelnden als Verräumlichung (...) in Form der folgenden Aspekte wieder gegenübertritt:

- Interaktions- und Machtstrukturen, in denen Individuen und Gruppen Räume herstellen und nutzen, in dem

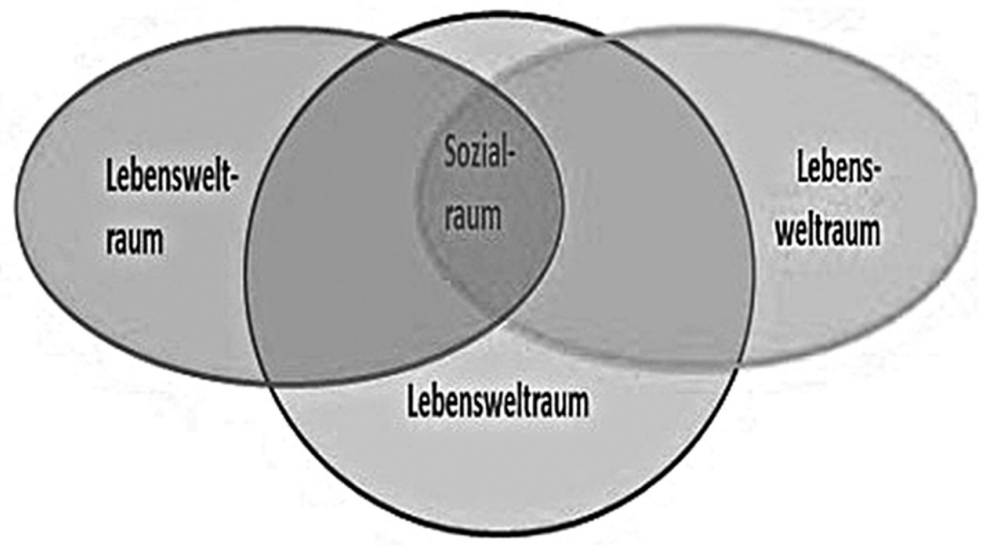

Abb. 1: Der Sozialraum entsteht vielfach als Teil verschiedener sich überlappender Räume von Lebenswelten. 
sie sich positionieren und andere ausschließen (...),

- Institutionalisierte normative Regulationssysteme, die durch Verrechtlichung regeln wie und durch wen Raum hergestellt und genutzt werden darf,

- Symbolsysteme, d. h. Bedeutungen, die räumliche Identifikation und Identifizierung schaffen" (Budde, Früchtel 2005: 238).

Mit dieser Bezugnahme auf Läpples (vgl. 1991) Ebenen des gesellschaftlichen Raumes und mit dem Verständnis der Verräumlichung auf diesen Ebenen als $\mathrm{Me}$ dium sowie Ergebnis individueller und organisatorischer Praktiken wird die Bearbeitung der Wechselbeziehungen zwischen physischem Ort und Sozialstruktur als Ansatzpunkt der Sozialraumorientierung verdeutlicht (vgl. Abb. 1).

Fachkräfte, die innerhalb eines sozialplanerisch definierten Raums, am Wohnort der Menschen erzieherische Hilfen erbringen, nehmen eine reflexive räumliche Haltung ein, wenn sie zwischen objektiv-materiellen Raumaspekten - als Ergebnis vorangegangener Platzierungen sozialer Güter sowie Menschen (vgl. Löw 2007: 89) - und den Folgen dieser Strukturen in den individuell konstruierten und sich überschneidenden Lebenswelträumen vermitteln (vgl. Abb. 2). Die Unterscheidung zwischen Lebenswelträumen und Sozialräumen ist eine Differenz, die auf Lewin (1963: 99) zurückgeht, der vom "Lebensraum « in Abgrenzung zur »Sozialen Welt« spricht.

Dass diese Vermittlung "nur « in sozialplanerisch definierten Räumen, stattfindet und nicht für jeden individuellen Lebensweltraum, hat pragmatische Gründe. Die Vielzahl der subjektiv konstruierten Lebenswelträume, die noch dazu verinselt sind, verhindert dieses Unterfangen. Ich spreche von Planungsräumen, weil diese "geographische Regionen (...) als Steuerungsgrößen « der Kinder- und Jugendhilfe keine Sozialräume sind, »auch wenn sie oft so genannt werden " (Budde et al. 2007: 256). "Während die historisch gewachsenen Gebiete mit kultureller Eigenart und Identität zwischen 5.000 und 20.000 Einwohnern variieren, umfassen die großstädtischen Bezirke zwischen 50.000 bis zu 80.000 Einwohner.« (Fehren 2008: 155) Somit entsteht das Dilemma der Inkongruenz zwischen einer gewissen Mindestgrö-

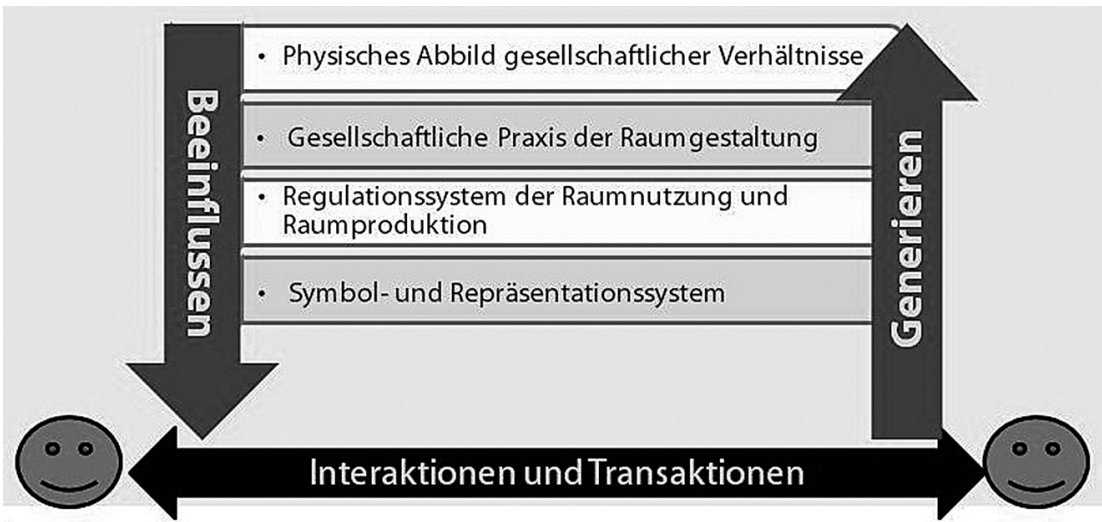

Abb. 2: Die Bildung von Planungsräumen muss verschiedene Aspekte berücksichtigen. ße von Verwaltungsraumeinheiten für Steuerungsprozesse und den natürlich gewachsenen Sozialräumen als Ergebnis sich überlappender Lebenswelträume.

Es lässt sich konstruktiv bearbeiten, in dem sich Soziale Arbeit »in ihrer Raumorientierung an die größeren Stadtbezirke (und im ländlichen Raum an die größeren Gemeinden, M. N.) als Bezugsgröße « anlehnt und »dann die meist kleineren bzw. kleinräumigeren Communities innerhalb dieses größeren Bezugsrahmens « (Fehren 2008: 156) bearbeitet (vgl. Abb. $3)$. Voraussetzung dafür ist eine $\mathrm{Zu}$ schneidung von Planungsräumen, die die Lebensweltraumüberlappungen nicht durchtrennt, so dass ein von der Bürokratie definierter Raum »mehrere solcher Überlappungen enthält, auf die sich die Fachkräfte dann jeweils nach Bedarf in ihren Schwerpunktsetzungen beziehen können« (Hinte 2005: 549).

\section{Sozialraumanalysen zur Schneidung von Planungsräumen}

In Berlin wurden die Ortsteile der Bezirke als Planungsräume zur Steuerung der Kinder- und Jugendhilfe ausgewählt (vgl. Moser, Seidel-Schäfer 2007: 65). Eine weitere Möglichkeit bietet die Zusammenfassung gewachsener Sozialräume - wie den Gemeinden eines Landkreises - zu einem Planungsraum, die beispielsweise im Landkreis Ravensburg genutzt wurde (vgl. Gutemann, Goller-Martin 2007: 90).

Planungsräume lassen sich auch sozialraumanalytisch anhand sozialstatistischer Merkmale bilden, die in Korrelati-

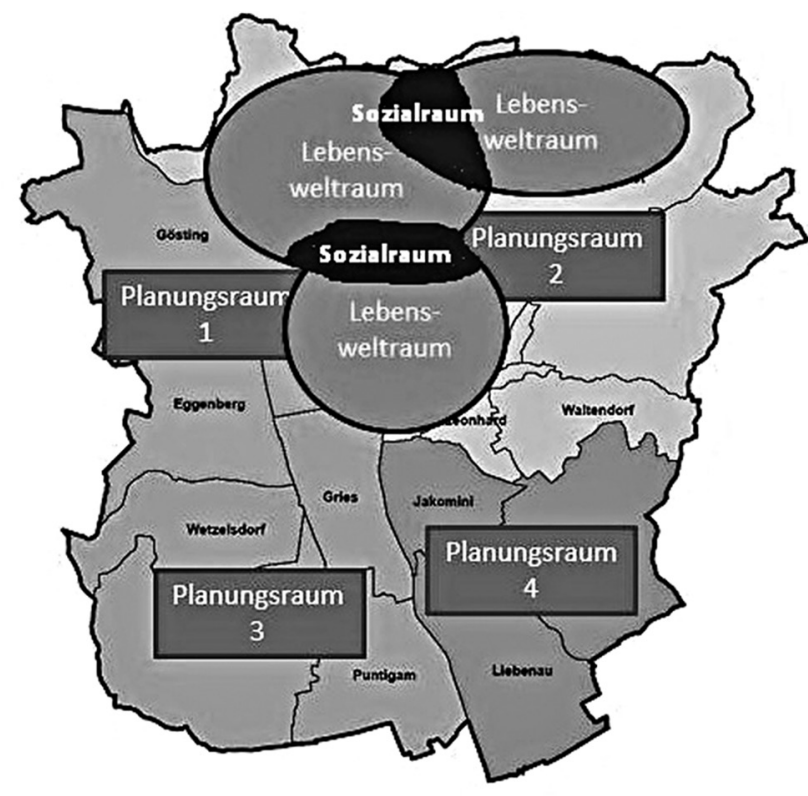

Abb. 3: Planungs-, Sozial- und Lebenswelträume stehen in Wechselbeziehung zueinander, wie die Aufteilung der österreichischen Stadt Graz zeigt. 
on zum Bedarf an erzieherischer Hilfen stehen. Für dieses bivariate Verfahren können die vorhandenen kommunalen Daten bezüglich Einwohnerzahl, Jugendlichen unter 21 Jahren sowie der Anzahl und der Art erbrachter Hilfeleistungen genutzt werden, wie beispielsweise in Rosenheim (vgl. Rose 2010: 29).

Um Geld und Personal für erzieherische Hilfen planungsräumlich zu steuern, kann die Faktorenanalyse genutzt werden. Mit diesem multivariaten Verfahren werden kleinräumige sozialstatistische Daten verdichtet, um die Planstellen des öffentlichen Kostenträgers in den Planungsräumen - ausgehend von Belastungsfaktoren - zu verteilen.

\section{Erfassung von Sozialräumen innerhalb von Planungsräumen}

Neben dem Vergleich kleinräumiger quantitativer statistischen Daten, bezogen auf einen Ortsteil oder ein Quartier des jeweiligen Planungsraumes, sollten für eine adäquate Erfassung natürlich gewachsener Sozialräume mit ihren infrastrukturellen Ressourcen und Defiziten unbedingt die subjektiven Einschätzungen junger Menschen eingeholt werden.

Ist eine qualitative Befragung aller Bewohner eines Planungsraumes zu diesen Einschätzungen möglich, ist das der »Königsweg «. Bei Planungsräumen mit 50.000 bis 80.000 Einwohnern wird eine solche Erhebung leider in den seltensten Fällen umsetzbar sein. Realistischer und wichtiger ist es, dass die Fachkräfte der Kostenträger und Leistungserbringer im Rahmen ihrer fallunspezifischen Arbeit von gewachsenen Sozialräumen innerhalb ihres Planungsraumes erfahren.

Fallunspezifische Arbeit findet regelmäßig statt, damit die Fachkräfte der Kosten- und Leistungsträger von Entwicklungen in ihrem Planungsraum erfahren, die zu veränderten Bedarfen und Ressourcenausstattungen in den Sozialräumen führen. Fallunspezifische Ressourcen werden in eine Ressourcenkartei übertragen.

\section{Erfassung individueller und gruppendynamisch vorgenommener Konstruktion von Lebenswelträumen}

Die Erfassung der Lebenswelträume von Empfängern erzieherischer Hilfen ist für die Fallarbeit in einem Planungsraum einerseits relevant, weil sich erst aus deren Überlappung ein Sozialraum bildet, in dem sich beispielsweise fallübergreifende Problemlagen abbilden, an denen mit fallübergreifenden Projekten angesetzt werden kann.

Andererseits kann nur durch die konkrete Aufschlüsselung jener Lebenswelträume, die sich zu einem Sozialraum überlappen, von bestehenden oder potenziellen solidarischen Austauschbeziehungen zwischen den Menschen in diesen Sozialräumen Kenntnis erlangt werden. Nicht zuletzt ist eine Analyse von sich überlappenden Lebenswelträumen notwendig, um erzieherische Hilfen an dem vielbeschworenen »sozialen Umfeld « $(\mathbb{S} 27$, Abs. 2, SGB VIII) junger Menschen auszurichten.

Hier besteht großes Potenzial in der Zusammenarbeit der Kinder- und Jugendhilfe mit der offenen Kinder- und Jugendarbeit (vgl. Deinet 2006: 526). Dafür bilden die "Sozialraumteams" aus dem Bereich der erzieherischen Hilfen und die »sozialräumlichen Gremien « der offenen Kinder- und Jugendarbeit eine ideale Schnittstelle (vgl. Deinet 2006: 524).

Mit ihrem Fundus qualitativer, lebensweltraumanalytischer Methoden (vgl. Deinet 2009) verfügt eine sozialräumlich orientierte Kinder- und Jugendarbeit über immenses Wissen über die Lebenswelträume junger Menschen. Diese Informationen sind für eine lebensweltraumsensible sozialraumorientierte Erbringung erzieherischer Hilfen relevant, weil die Fallarbeiter von den subjektiv und jeweils situationsspezifisch konstruierten Lebenswelträumen, die sich gruppendynamisch zu Sozialräumen überlappen können, leichter Kenntnis erlangen können.

Eine Wissensübermittlung ist möglich, indem die Mitarbeiter der Erziehungshilfe an der Interpretation lebensweltraumanalytisch gewonnener Informationen beteiligt werden und die Interpretationsergebnisse bei der Planung und Erbringung erzieherischer Hilfen berücksichtigen und die Fachkräfte der offenen Kinder- und Jugendarbeit an der multiperspektivischen, kollegialen Fallberatung im Sozialraumteam beteiligt werden (vgl. Deinet 2006: 525).

\section{Literatur}

Budde, W.; Früchtel, F.; Cyprian, G. (2007): Sozialer Raum und Soziale Arbeit. Textbook: Theoretische Grundlagen. Wiesbaden: VS Verlag.

Deinet, U. (2009): Sozialräumliche Jugendarbeit. Grundlagen, Methoden und Praxiskonzepte. 2. Auflage. Wiesbaden: VS Verlag. Deinet, U. (2006): Sozialräumliche Kooperation zwischen Offener Kinder- und Jugendarbeit und den Hilfen zur Erziehung. In: Deutsche Jugend, Heft 12. S. 519-526.

Fehren, 0. (2008): Wer organisiert das Gemeinwesen? Zivilgesellschaftliche Perspektiven Sozialer Arbeit als intermediärer Instanz. Berlin: Sigma.

Gutemann, K; Goller-Martin, S. (2007): Sozialraumorientierte Jugendhilfe im Landkreis Ravensburg. In: Verein für Kommunalwis senschaften e. V. (Hg.): Sozialraumorientiert Umbau der Hilfen zur Erziehung: Positive Effekte, Risiken und Nebenwirkungen.

Band 2. S.: 87-99.

Hinte, W.; Treeß, H. (2007): Sozialraumorientierung in der Jugendhilfe. Theoretische Grundlagen, Handlungsprinzipien und Praxisbeispiele einer kooperativ-integrativen Pädagogik. Weinheim, Basel und München: Juventa.

Hinte, W. (2005): Diskussionsbeitrag Gemeinwesenarbeit. In: Kessl, F.; Reutlinger, C.; Maurer, S.; Frey, O. (Hg.): Handbuch Sozialraum. S.: 548-554.

Budde, W.; Früchtel, F. (2005): Sozialraumorientierte Soziale Arbeit - ein Modell zwischen Lebenswelt und Steuerung. In: NDV, 7/2005, S.: 238-242 und 8/2005, S.: 287-292.

Läpple, D. (1991): Essay über den Raum. Für ein gesellschaftswissenschaftliches Raumkonzept. In: Häußermann, H., u. a. (Hg.): Stadt und Raum. Soziologische Analysen. Pfaffenweiler. Lewin, K. (1963): Feldtheorie in den Sozialwissenschaften. Ausgewählte theoretische Schriften. Berlin und Stuttgart: Verlag Hans Huber. Löw, M. (2007): Zwischen Handeln und Struktur. In: Kessl, F.; Otto, H. U. (Hg.) : Territorialisierung des Sozialen. Regieren über lokale Nahräume. Opladen \& Farmington Hills: Verlag Barbara Budrich. S.: 81-100.

Moser, T.; Seidel-Schäfer, G. (2007): Verschränkung von Fach- und Finanzcontrolling am Beispiel eines Berliner Bezirks: "Jugendhilfe ist nicht steuerbar!« - Oder doch?. In: Verein für Kommunalwissenschaften e. V. (Hg.). S.: 63-70. Rose, G. (2010): Projektarchitektur in Rosenheim: Erste Schritte - Meilensteine - Stolpersteine. In: Pichelmeier, W.; Rose, G. (Hg.): Sozialraumorientierte Jugendhilfe in der Praxis. Handreichung für kommunale Entscheidungsträger am Beispiel der Stadt Rosenheim. Rosenheim: Kommunal-Verlag. S.: 27-33. 